\title{
Tidal propagation in an oceanic island with sloping beaches
}

\author{
Y.-C. Chang ${ }^{1}$, D.-S. Jeng ${ }^{2,3}$, and H.-D. Yeh ${ }^{1}$ \\ ${ }^{1}$ Institute of Environmental Engineering, National Chiao Tung University, Hsinchu, Taiwan \\ ${ }^{2}$ Department of Civil Engineering, Shanghai Jiao Tong University, Shanghai 200240, China \\ ${ }^{3}$ Division of Civil Engineering, University of Dundee, Scotland, DD1, 4HN, UK
}

Received: 31 December 2009 - Published in Hydrol. Earth Syst. Sci. Discuss.: 22 February 2010

Revised: 4 June 2010 - Accepted: 10 June 2010 - Published: 26 July 2010

\begin{abstract}
In this study, a new analytical solution for describing the tide-induced groundwater fluctuations in oceanic islands with finite length and different slopes of the beaches is developed. Unlike previous solutions, the present solution is not only applicable for a semi-infinite coastal aquifer, but also for an oceanic island with finite length and different sloping beaches. The solution can be used to investigate the effect of higher-order components and beach slopes on the water table fluctuations. The results demonstrate the effect of higher-order components increases with the shallow water parameter or amplitude parameter and the water table level increases as beach slopes decrease.
\end{abstract}

\section{Introduction}

Groundwater near the ocean usually fluctuates with the tides, which will significantly affect the costal processes such as saltwater intrusions, beach sediment transportations, chemical transformations, and biological activities. To understand and manage the behavior of coastal aquifers, it is required to accurately predict the dynamic groundwater hydraulics. Most studies for groundwater fluctuations in costal aquifers are based on Boussinesq equation with Dupuit assumption (Freeze and Cherry, 1979). Dagan (1967) first solved the non-linear governing equation and approximated the solution by an expansion based on a shallow flow approximation. His solution is applicable when the oscillation of the groundwater motion is small compared with the mean water level. Parlange et al. (1984) extended the work of Dagan (1967) and used a perturbation technique to derive an approximate solution of the non-linear equation. Their solution demonstrates that the nonlinear effects on tidal propagation are not

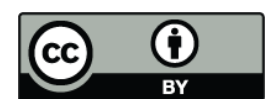

Correspondence to: $\mathrm{H}$.-D. Yeh

(hdyeh@mail.nctu.edu.tw) negligible and the omission of the effects may lead to a significant error in predicting water table elevation. Previous studies for water table fluctuations in costal aquifers considered the case of vertical beach. For more realistic case of beach slopes, Nielsen (1990) considered a movable shoreline boundary condition and derived an analytical solution for groundwater fluctuations in costal aquifers with a sloping beach. Li et al. (2000) further overcame the inconsistency of the boundary condition in Nielsen's solution and utilized the same parameter in Nielsen (1990) to develop a solution for the problem using a concept of moving boundary. Since the slope of the beach was included in the perturbation parameter in both models, their models may be applicable to a certain range of the beach slopes. Based on the reason mentioned above, Teo et al. (2003) used two perturbation parameters, shallow water parameter and amplitude parameter, to derive a higher-order solution for the tide-induced water table fluctuations in coastal aquifers to a sloping beach. They considered the costal aquifers to with infinite extension in horizontal direction; however, for oceanic islands, the horizontal domain is finite and the beach slopes may be different in both sides of the oceanic island.

Considering the oceanic island in coastal area, Jiao et al. (2001) investigated impact of land reclamation on groundwater flow systems. They derived steady-state solutions to show how the groundwater level, groundwater divide and submarine groundwater discharge would change with land reclamation near coastal aquifer. Hu et al. (2008) extended the work in Jiao et al. (2001) to develop the transient solution for groundwater flow induced by land reclamation in oceanic land. Rotzoll et al. (2008) presented an analytical solution derived from one-dimensional confined flow equation for hydraulic head distribution in a finite-length and asynchronous dual-tide aquifer. They analyzed the tidal responses in the unconfined central Maui Aquifer and estimated the hydraulic parameters in the study area. Sun et al. (2008) considered an island aquifer system comprising a confined aquifer and an

Published by Copernicus Publications on behalf of the European Geosciences Union. 


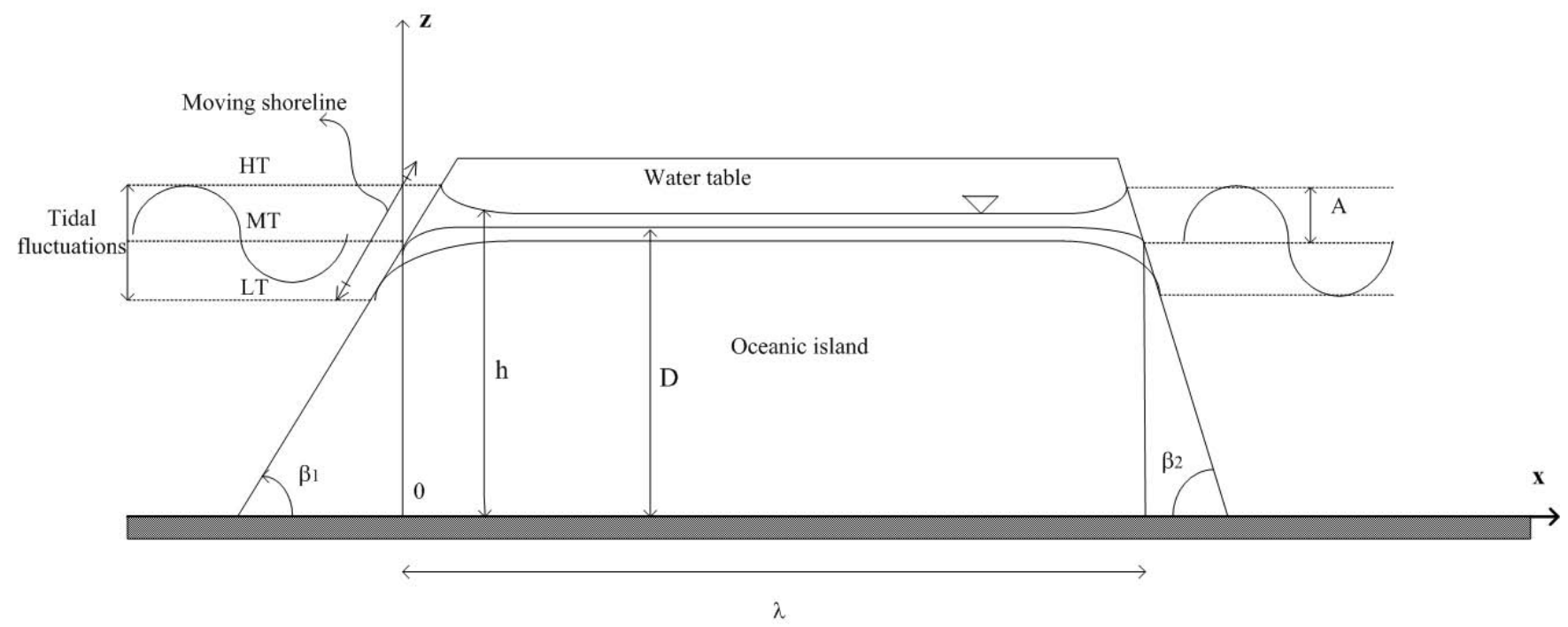

Fig. 1. The profile of tidal water table fluctuations in an oceanic island with sloping beaches.

overlying semipermeable layer. They derived an analytical solution for groundwater head response in the island aquifer system subject to dual-tide and compared the solution with the existing analytical solutions.

Motivated by the literatures mentioned above, the objective of this study is to develop a model for describing the tideinduced groundwater fluctuations in unconfined aquifers, which, to the best of our knowledge, have never been presented for the case of oceanic islands with dual-tide and different slopes of the beaches.

\section{Mathematical model and analytical solution}

\subsection{Mathematical statement}

The configuration of tidal induced groundwater flow is shown in Fig. 1. Assuming the fluid is incompressible and inviscid, the potential head $\phi$ satisfies the Laplace's equation

$\phi_{x x}+\phi_{z z}=0,0 \leq z \leq h(x, t)$

where $h(x, t)[L]$ is the total tidal induced water table height. The tidal oscillations cause two moving boundary conditions at the coasts which can be expressed as

$h\left(x_{0}(t), t\right)=D(1+\alpha \cos \omega t)$ on $x_{0}(t)=A \cot \beta_{1} \cos \omega t$

and

$h\left(\lambda-x_{\lambda}(t), t\right)=D(1+\alpha \cos \omega t)$ on $x_{\lambda}(t)=A \cot \beta_{2} \cos \omega t$

for left-hand side (LHS) and right-hand side (RHS) of the oceanic island, respectively. The length of the oceanic island is denoted as $\lambda$ in Fig. 1.

The $x_{0}(t)$ and $x_{\lambda}(t)$ are the horizontal extent of the tidal variation at the sloping beaches, the beach angles for LHS and RHS of the island are, respectively, denoted as $\beta_{1}$ and $\beta_{2}$. The dimensionless amplitude parameter $\alpha=A / D$ represents the ratio of the maximum tidal amplitude, $A[\mathrm{~L}]$, to the average water table height, $D[L]$, and $\omega\left[T^{-1}\right]$ is the tidal frequency. At the bottom of the island, the boundary condition is

$$
\frac{\partial \phi}{\partial z}=0 \text { at } z=0
$$

The boundary at the water table can be represented as

$\phi=h$ at $z=h$

and the flow at the water table is modeled by the following equation (Batu, 1998)

$n_{e} \frac{\partial \phi}{\partial t}=K\left[\left(\frac{\partial \phi}{\partial x}\right)^{2}+\left(\frac{\partial \phi}{\partial z}\right)^{2}\right]-K \frac{\partial \phi}{\partial z}$ at $z=h$

where $n_{e}$ is the effective porosity and $K$ is the saturated hydraulic conductivity. Both parameters are assumed constants.

\subsection{Perturbation approach}

The governing equation and boundary conditions are rewritten in dimensionless forms using the following nondimensional variables

$X=\frac{x}{L}, \quad Z=\frac{z}{D}, \quad H=\frac{h}{D}, \quad \Phi=\frac{\phi}{D}$,

$\varepsilon=\frac{D}{L}, \quad \lambda^{*}=\frac{\lambda}{L}, \quad$ and $T=\omega t$

where $L=\sqrt{\frac{2 K D}{n_{e} \omega}}$ is a decay length scale of water table fluctuations and $\varepsilon$ is defined as the shallow water parameter. The governing Eq. (1) becomes

$\Phi_{Z Z}=-\varepsilon^{2} \Phi_{X X}$ 
and the boundary conditions (Eqs. 4-6) lead to

$\Phi_{Z}=0$ at $Z=0$

$\Phi=H$ at $Z=H$

and

$2 \varepsilon^{2} \Phi_{T}=\varepsilon^{2} \Phi_{X}^{2}+\Phi_{Z}^{2}-\Phi_{Z}$ at $Z=H$

where $\Phi_{X}, \Phi_{Z}$ and $\Phi_{T}$ represent the first derivatives of $\Phi$ with respect to $X, Z$ and $T$, respectively. In addition, $\Phi_{X X}$ and $\Phi_{Z Z}$ represent the second derivatives of $\Phi$ with respect to $X$ and $Z$, respectively.

The boundary conditions in Eqs. (2) and (3), respectively, becomes

$H\left(X_{0}(T), T\right)=1+\alpha \cos T$ on

$X_{0}(T)=\alpha \varepsilon \cot \beta_{1} \cos T$

and

$H\left(\lambda^{*}-X_{\lambda}(T), T\right)=1+\alpha \cos T$ on

$X_{\lambda}(T)=\alpha \varepsilon \cot \beta_{2} \cos T$

By introducing the new variables (Li et al., 2000; Teo et al., 2003)

$X_{1}=X-X_{0}(T)$ and $T_{1}=T$

Then

$\frac{\partial f}{\partial T}=\frac{\partial f}{\partial T_{1}}+\frac{\partial f}{\partial X_{1}} \frac{\partial X_{1}}{\partial T}=\frac{\partial f}{\partial T_{1}}+\alpha \varepsilon \cot \beta_{1} \sin T_{1} \frac{\partial f}{\partial X_{1}}$

where $f$ is a dependent variable such as $\Phi$ and $H$. Equations (12) and (13) can be, respectively, transformed to

$H\left(X_{L}\left(T_{1}\right), T_{1}\right)=1+\alpha \cos T_{1}$ on $X_{L}\left(T_{1}\right)=0$

and

$H\left(X_{R}\left(T_{1}\right), T_{1}\right)=1+\alpha \cos T_{1}$ on $X_{R}\left(T_{1}\right)$

$=\lambda^{*}-\alpha \varepsilon \cot \beta_{2} \cos T_{1}-\alpha \varepsilon \cot \beta_{1} \cos T_{1}$

where $X_{L}$ and $X_{R}$ denote the moving boundary on the LHS and RHS of the island.

Assuming that the potential head $\Phi$ and water table level $H$ can be expanded in powers of $\varepsilon$, respectively, as

$\Phi(X, Z, T)=\sum_{n=0}^{\infty} \varepsilon^{n} \Phi_{n}(X, Z, T)$

and

$H(X, T)=\sum_{n=0}^{\infty} \varepsilon^{n} H_{n}(X, T)$
The detail of derivation for Eq. (8) with boundary conditions in Eqs. (9) and (10) is listed in Appendix A and the results up to second-order are

$O\left(\varepsilon^{0}\right): 2 H_{0 T_{1}}=\left(H_{0} H_{0 X_{1}}\right)_{X_{1}}$

$H_{0}\left(0, T_{1}\right)=H_{0}\left(X_{R}, T_{1}\right)=1+\alpha \cos T_{1}$

$O\left(\varepsilon^{1}\right): \quad 2\left(H_{1 T_{1}}+\alpha \cot \beta_{1} \sin T_{1} H_{0 X_{1}}\right)=\left(H_{0} H_{1}\right)_{X_{1} X_{1}}$

$H_{1}\left(0, T_{1}\right)=H_{1}\left(X_{R}, T_{1}\right)=0$

$O\left(\varepsilon^{2}\right): 2\left(H_{2 T_{1}}+\alpha \cot \beta_{1} \sin T_{1} H_{1 X_{1}}\right)=$

$\frac{1}{2}\left(H_{1}^{2}\right)_{X_{1} X_{1}}+\left(H_{0} H_{2}\right)_{X_{1} X_{1}}+\frac{1}{3}\left(H_{0}^{3} H_{0 X_{1} X_{1}}\right)_{X_{1} X_{1}}$

$H_{2}\left(0, T_{1}\right)=H_{2}\left(X_{R}, T_{1}\right)=0$

\subsubsection{Zero-order $\left(O\left(\varepsilon^{0}\right)\right)$ approximation:}

The perturbation expansion of $H_{0}$ in power of $\alpha$ can be expressed as

$H_{0}=1+\sum_{n=1}^{\infty} \alpha^{n} H_{0 n}$

Equation (19a) and (b) can be expanded in different order of $\alpha$ as:

$O\left(\varepsilon^{0} \alpha^{1}\right): 2 H_{01 T_{1}}=H_{01 X_{1} X_{1}}$

$H_{01}\left(0, T_{1}\right)=H_{01}\left(X_{R}, T_{1}\right)=\cos T_{1}$

$O\left(\varepsilon^{0} \alpha^{2}\right): 2 H_{02 T_{1}}=H_{02 X_{1} X_{1}}+\left(H_{01} H_{01 X_{1}}\right)_{X_{1}}$

$H_{02}\left(0, T_{1}\right)=H_{02}\left(X_{R}, T_{1}\right)=0$

The derivation for the solutions of Eq. (23) is given in Appendix $\mathrm{B}$ and the result is

$H_{01}=e^{-X_{1}} \cos \left(T_{1}-X_{1}\right)$

$+a_{1}\left[e^{X_{1}} \cos \left(T_{1}+X_{1}\right)-e^{-X_{1}} \cos \left(T_{1}-X_{1}\right)\right]$

$+a_{2}\left[e^{X_{1}} \sin \left(T_{1}+X_{1}\right)-e^{-X_{1}} \sin \left(T_{1}-X_{1}\right)\right]$

Similarly, the solution of Eq. (24) can be obtained as

$H_{02}=-\frac{1}{4}\left(\delta_{11} e^{-2 X_{1}}+\delta_{12} e^{2 X_{1}}+2 \delta_{13} \cos 2 X_{1}+2 \delta_{14} \sin 2 X_{1}\right)$

$-\frac{1}{2} e^{-2 X_{1}}\left[\delta_{16} \cos 2\left(T_{1}-X_{1}\right)+\delta_{15} \sin 2\left(T_{1}-X_{1}\right)\right]$

$-\frac{1}{2} e^{2 X_{1}}\left[\delta_{18} \cos 2\left(T_{1}+X_{1}\right)+\delta_{17} \sin 2\left(T_{1}+X_{1}\right)\right]$

$+b_{1} X_{1}+b_{2}+c_{1} e^{\sqrt{2} X_{1}} \cos \left(2 T_{1}+\sqrt{2} X_{1}\right)$

$+c_{2} e^{\sqrt{2} X_{1}} \sin \left(2 T_{1}+\sqrt{2} X_{1}\right)$

$+c_{3} e^{-\sqrt{2} X_{1}} \cos \left(2 T_{1}-\sqrt{2} X_{1}\right)$

$-c_{4} e^{-\sqrt{2} X_{1}} \sin \left(2 T_{1}-\sqrt{2} X_{1}\right)$ 

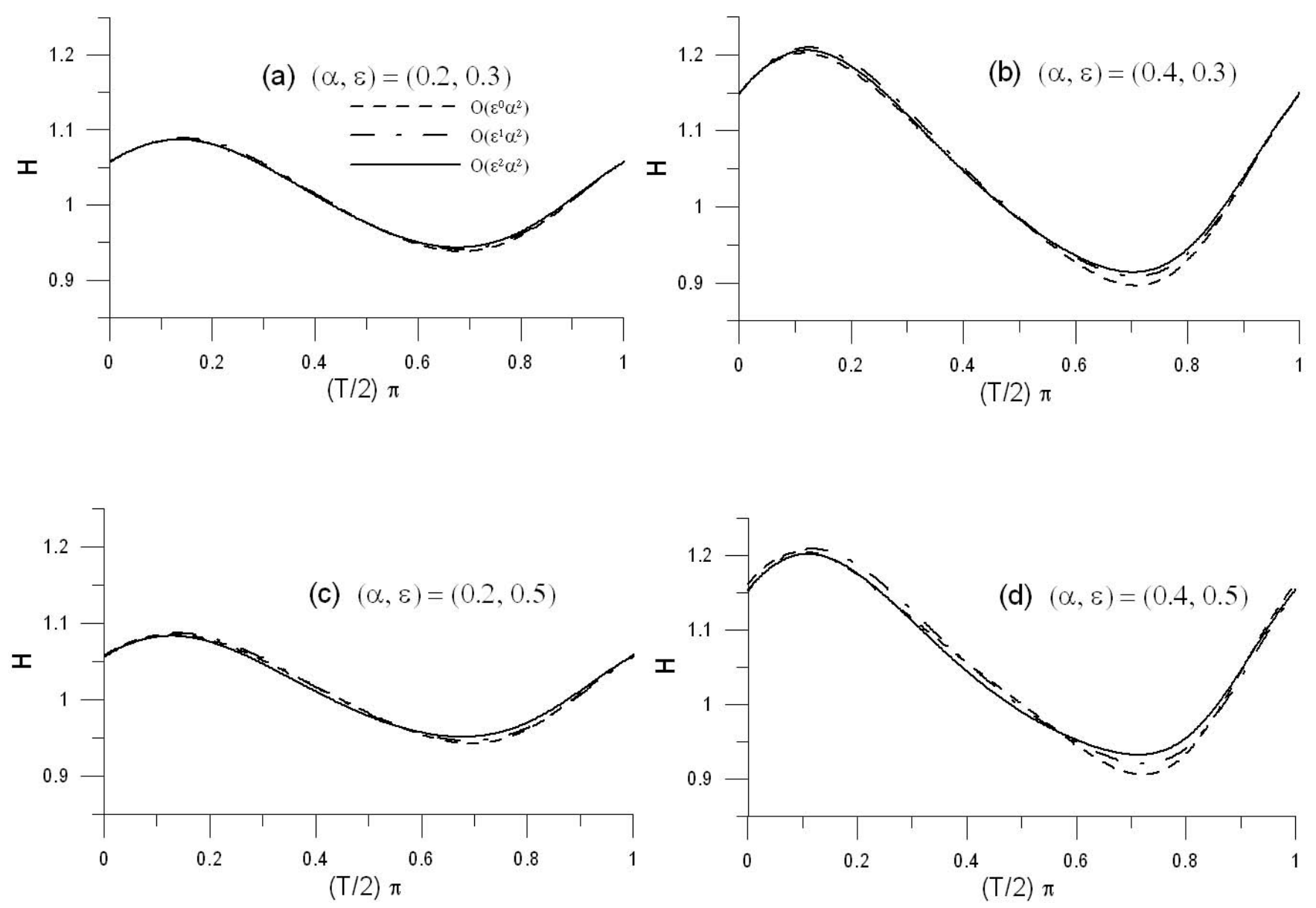

Fig. 2. Distribution of water table level $(H)$ versus time $(T / 2 \phi)$ in an oceanic island at $X=1$ with beach slopes $\beta_{1}=\beta_{2}=45^{\circ}$.

The coefficients $a_{1}, a_{2}, \delta_{11}, \delta_{12}, \delta_{13}, \delta_{14}, b_{1}, b_{2}, c_{1}, c_{2}, c_{3}$ and $c_{4}$ in Eqs. (22) and (26), defined in Table 1, are obtained from the formula manipulation package of Mathematica 6 (Wolfram Research, Inc., 2007). For the case that the costal aquifer has a half domain with $\lambda \rightarrow \infty, a_{1}$ and $a_{2}$ approach to zero. Equations (25) and (26) are identical to the solutions in Teo et al. (2003) when $X_{1}$ is sufficiently small for zero-order approximation in $\varepsilon$ for order $\alpha^{1}$ and $\alpha^{2}$, respectively.

\subsubsection{First-order $\left(O\left(\varepsilon^{1}\right)\right)$ approximation:}

For $O\left(\varepsilon^{1}\right), H_{1}$ can be expanded as

$H_{1}=\sum_{n=1}^{\infty} \alpha^{n} H_{1 n}$

The equation and boundary conditions can be arranged as

$O\left(\varepsilon^{1} \alpha^{1}\right): 2 H_{11 T_{1}}=H_{11 X_{1} X_{1}}$

$H_{11}\left(0, T_{1}\right)=H_{11}\left(X_{R}, T_{1}\right)=0$

$O\left(\varepsilon^{1} \alpha^{2}\right): 2 H_{12 T_{1}}+2 \cot \beta_{1} \sin T_{1} H_{01 X_{1}}$

$=H_{12 X_{1} X_{1}}+\left(H_{01} H_{11}\right)_{X_{1} X_{1}}$
$H_{12}\left(0, T_{1}\right)=H_{12}\left(X_{R}, T_{1}\right)=0$

The solution for Eq. (28) is $H_{11}=0$. Substituting $H_{01}$ in Eqs. (22) into (29), the solution of Eq. (29) is

$$
\begin{aligned}
& H_{12}=\frac{1}{2} \cot \beta_{1}\left\{\left[e^{-X_{1}}\left(\delta_{21} \cos X_{1}-\delta_{22} \sin X_{1}\right)\right.\right. \\
& \left.+e^{X_{1}}\left(\delta_{23} \cos X_{1}+\delta_{24} \sin X_{1}\right)\right]+\left(d_{1} X_{1}+d_{2}\right) \\
& +\left[e^{-X_{1}}\left(\delta_{21} \cos \left(2 T_{1}-X_{1}\right)+\delta_{22} \sin \left(2 T_{1}-X_{1}\right)\right)\right. \\
& \left.+e^{X_{1}}\left(\delta_{23} \cos \left(2 T_{1}+X_{1}\right)+\delta_{24} \sin \left(2 T_{1}+X_{1}\right)\right)\right] \\
& 2\left[e^{\sqrt{2} X_{1}}\left(f_{1} \cos \left(2 T_{1}+\sqrt{2} X_{1}\right)+f_{2} \sin \left(2 T_{1}+\sqrt{2} X_{1}\right)\right)\right. \\
& \left.\left.+e^{-\sqrt{2} X_{1}}\left(f_{3} \cos \left(2 T_{1}-\sqrt{2} X_{1}\right)+f_{4} \sin \left(2 T_{1}-\sqrt{2} X_{1}\right)\right)\right]\right\}
\end{aligned}
$$

The coefficients $\delta_{21}, \delta_{22}, \delta_{23}, \delta_{24}, d_{1}, d_{2}, f_{1}, f_{2}, f_{3}$ and $f_{4}$ in Eq. (30), defined in Table 1, are obtained from the formula manipulation package of Mathematica 6 (Wolfram Re- 
Table 1. Definition of coefficients.

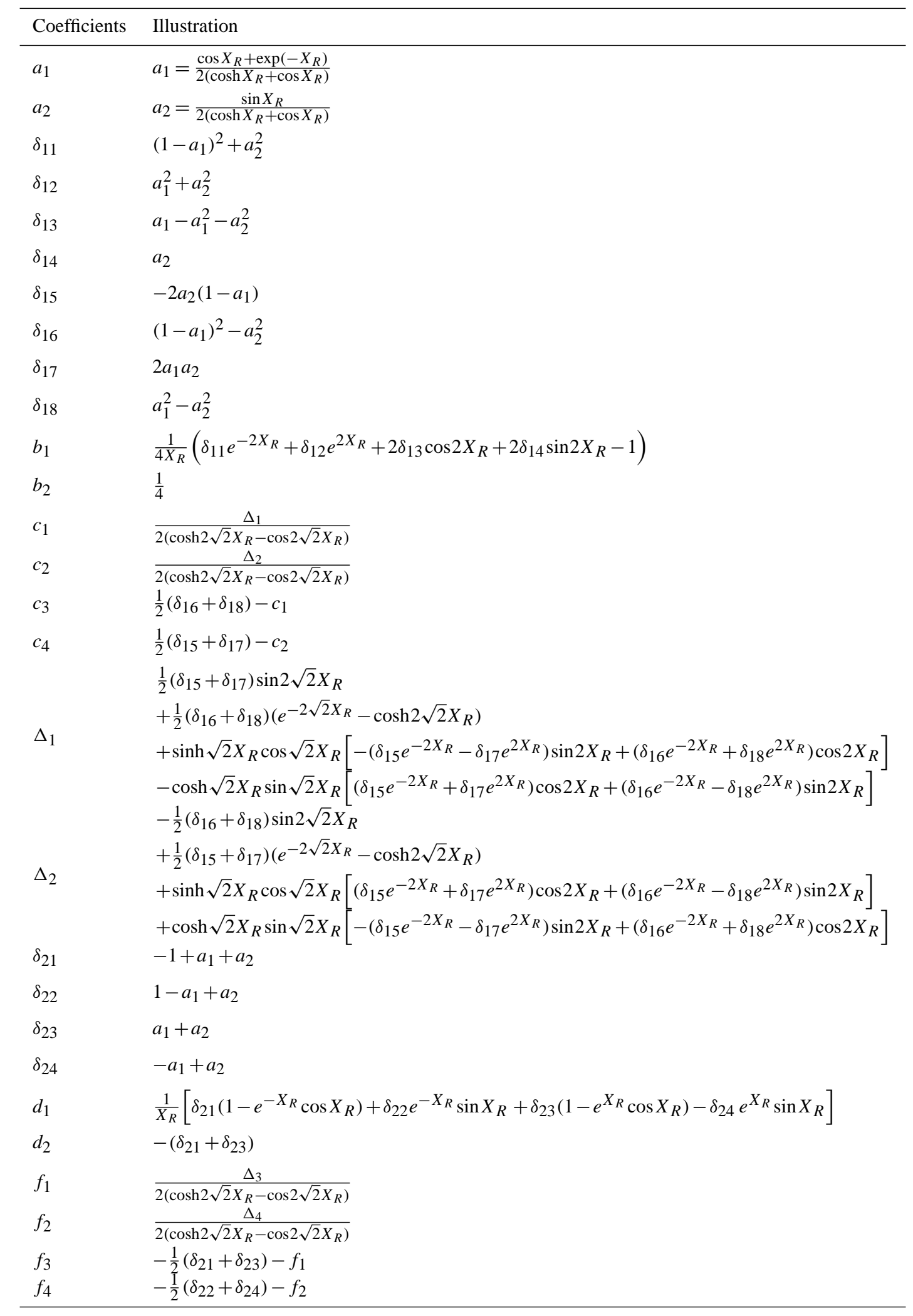


Table 1. Continued.

\begin{tabular}{|c|c|}
\hline Coefficients & Illustration \\
\hline$\Delta_{3}$ & $\begin{array}{l}-\frac{1}{2}\left(\delta_{22}+\delta_{24}\right) \sin 2 \sqrt{2} X_{R} \\
-\frac{1}{2}\left(\delta_{21}+\delta_{23}\right)\left(e^{-2 \sqrt{2} X_{R}}-\cosh 2 \sqrt{2} X_{R}\right) \\
+\sinh \sqrt{2} X_{R} \cos \sqrt{2} X_{R}\left[\left(\delta_{22} e^{-2 X_{R}}-\delta_{24} e^{2 X_{R}}\right) \sin X_{R}-\left(\delta_{21} e^{-2 X_{R}}+\delta_{23} e^{2 X_{R}}\right) \cos X_{R}\right] \\
+\cosh \sqrt{2} X_{R} \sin \sqrt{2} X_{R}\left[\left(\delta_{22} e^{-2 X_{R}}+\delta_{24} e^{2 X_{R}}\right) \cos X_{R}+\left(\delta_{21} e^{-2 X_{R}}-\delta_{23} e^{2 X_{R}}\right) \sin X_{R}\right]\end{array}$ \\
\hline$\Delta_{4}$ & $\begin{array}{l}\frac{1}{2}\left(\delta_{21}+\delta_{23}\right) \sin 2 \sqrt{2} X_{R} \\
-\frac{1}{2}\left(\delta_{22}+\delta_{24}\right)\left(e^{-2 \sqrt{2} X_{R}}-\cosh 2 \sqrt{2} X_{R}\right) \\
-\sinh \sqrt{2} X_{R} \cos \sqrt{2} X_{R}\left[\left(\delta_{22} e^{-2 X_{R}}+\delta_{24} e^{2 X_{R}}\right) \cos X_{R}+\left(\delta_{21} e^{-2 X_{R}}-\delta_{23} e^{2 X_{R}}\right) \sin X_{R}\right]\end{array}$ \\
\hline$g_{1}$ & $\begin{array}{l}+\cosh \sqrt{2} X_{R} \sin \sqrt{2} X_{R}\left[\left(\delta_{22} e^{-2 \Lambda R}-\delta_{24} e^{2 \Lambda}\right) \sin X_{R}-\left(\delta_{21} e^{-2 \Lambda_{R}}+\delta_{23} e^{2 \Lambda R}\right) \cos X_{R}\right] \\
\frac{\Delta_{5}}{3\left(\cosh 2 X_{R}-\cos 2 X_{R}\right)}\end{array}$ \\
\hline$g_{2}$ & $\frac{\Delta_{6}}{3\left(\cosh 2 X_{R}-\cos 2 X_{R}\right)}$ \\
\hline$g_{3}$ & $-g_{1}$ \\
\hline$g_{4}$ & $-g_{2}$ \\
\hline$\Delta_{5}$ & $X_{R}\left[\cos 2 X_{R}-\cosh 2 X_{R}-\left(1-2 a_{1}-2 a_{2}\right) \sin 2 X_{R}+\left(1-2 a_{1}+2 a_{2}\right) \sinh 2 X_{R}\right]$ \\
\hline$\Delta_{6}$ & $X_{R}\left[\cos 2 X_{R}-\cosh 2 X_{R}+\left(1-2 a_{1}+2 a_{2}\right) \sin 2 X_{R}+\left(1-2 a_{1}-2 a_{2}\right) \sinh 2 X_{R}\right]$ \\
\hline$k_{1}$ & $\frac{\Delta_{7}}{2\left(\cos \left(2 \sqrt{2} X_{R}\right)-\cosh \left(2 \sqrt{2} X_{R}\right)\right)\left(\cosh \sqrt{2} X_{R}+\sinh \sqrt{2} X_{R}\right)^{2}}$ \\
\hline$k_{2}$ & $\frac{\Delta_{8}}{2\left(\cos \left(2 \sqrt{2} X_{R}\right)-\cosh \left(2 \sqrt{2} X_{R}\right)\right)\left(\cosh \sqrt{2} X_{R}+\sinh \sqrt{2} X_{R}\right)^{2}}$ \\
\hline$k_{3}$ & $-k_{1}$ \\
\hline$k_{4}$ & $-k_{2}$ \\
\hline$\varphi_{1}$ & $\frac{1}{6}\left(2-4 a_{1}+2 a_{1}^{2}+4 a_{2}-4 a_{1} a_{2}-2 a_{2}^{2}\right)$ \\
\hline$\varphi_{2}$ & $\varphi_{1}$ \\
\hline$\varphi_{3}$ & $\frac{1}{6}\left(-2 a_{1}^{2}+4 a_{1} a_{2}+2 a_{2}^{2}\right)$ \\
\hline \multirow[t]{2}{*}{$\varphi_{4}$} & $\varphi_{3}$ \\
\hline & $\begin{array}{l}-\left(X_{R}\left(\cosh \left((-2+\sqrt{2}) X_{R}\right)+\sinh \left((-2+\sqrt{2}) X_{R}\right)\right)\left(\varphi_{1} \cos \left((-2+\sqrt{2}) X_{R}\right)\right.\right. \\
-\varphi_{3} \cos \left((-2+\sqrt{2}) X_{R}\right) \cosh \left(2(2+\sqrt{2}) X_{R}\right)+\varphi_{2} \sin \left((-2+\sqrt{2}) X_{R}\right) \\
+\varphi_{4} \sin \left((-2+\sqrt{2}) X_{R}\right) \cosh \left(2(2+\sqrt{2}) X_{R}\right)+\cosh \left(2 \sqrt{2} X_{R}\right)\left(-\varphi_{1} \cos \left((2+\sqrt{2}) X_{R}\right)\right.\end{array}$ \\
\hline \multirow[t]{4}{*}{$\Delta_{7}$} & $\left.+\varphi_{2} \sin \left((2+\sqrt{2}) X_{R}\right)\right)+\cosh \left(4 X_{R}\right)\left(\varphi_{3} \cos \left((2+\sqrt{2}) X_{R}\right)+\varphi_{4} \sin \left((2+\sqrt{2}) X_{R}\right)\right.$ \\
\hline & $+\varphi_{3} \cos \left((2+\sqrt{2}) X_{R}\right) \sinh \left(4 X_{R}\right)+\varphi_{4} \sin \left((2+\sqrt{2}) X_{R}\right) \sinh \left(4 X_{R}\right)$ \\
\hline & $-\varphi_{1} \cos \left((2+\sqrt{2}) X_{R}\right) \sinh \left(2 \sqrt{2} X_{R}\right)+\varphi_{2} \sin \left((2+\sqrt{2}) X_{R}\right) \sinh \left(2 \sqrt{2} X_{R}\right)$ \\
\hline & $\begin{array}{l}-\varphi_{3} \cos \left((-2+\sqrt{2}) X_{R}\right) \sinh \left(2(2+\sqrt{2}) X_{R}\right)+\varphi_{4} \sin \left((-2+\sqrt{2}) X_{R}\right) \sinh \left(2(2+\sqrt{2}) X_{R}\right) \\
-\left(X_{R}\left(\cosh \left((-2+\sqrt{2}) X_{R}\right)+\sinh \left((-2+\sqrt{2}) X_{R}\right)\right)\left(-\varphi_{2} \cos \left((-2+\sqrt{2}) X_{R}\right)\right.\right. \\
+\varphi_{4} \cos \left((-2+\sqrt{2}) X_{R}\right) \cosh \left(2(2+\sqrt{2}) X_{R}\right)+\varphi_{1} \sin \left((-2+\sqrt{2}) X_{R}\right) \\
+\varphi_{3} \sin \left((-2+\sqrt{2}) X_{R}\right) \cosh \left(2(2+\sqrt{2}) X_{R}\right)+\cosh \left(2 \sqrt{2} X_{R}\right)\left(\varphi_{2} \cos \left((2+\sqrt{2}) X_{R}\right)\right.\end{array}$ \\
\hline \multirow[t]{4}{*}{$\Delta_{8}$} & $\left.+\varphi_{1} \sin \left((2+\sqrt{2}) X_{R}\right)\right)+\cosh \left(4 X_{R}\right)\left(-\varphi_{4} \cos \left((2+\sqrt{2}) X_{R}\right)+\varphi_{3} \sin \left((2+\sqrt{2}) X_{R}\right)\right.$ \\
\hline & $+\varphi_{4} \cos \left((2+\sqrt{2}) X_{R}\right) \sinh \left(4 X_{R}\right)+\varphi_{3} \sin \left((2+\sqrt{2}) X_{R}\right) \sinh \left(4 X_{R}\right)$ \\
\hline & $+\varphi_{2} \cos \left((2+\sqrt{2}) X_{R}\right) \sinh \left(2 \sqrt{2} X_{R}\right)+\varphi_{1} \sin \left((2+\sqrt{2}) X_{R}\right) \sinh \left(2 \sqrt{2} X_{R}\right)$ \\
\hline & $+\varphi_{4} \cos \left((-2+\sqrt{2}) X_{R}\right) \sinh \left(2(2+\sqrt{2}) X_{R}\right)+\varphi_{3} \sin \left((-2+\sqrt{2}) X_{R}\right) \sinh \left(2(2+\sqrt{2}) X_{R}\right)$ \\
\hline
\end{tabular}



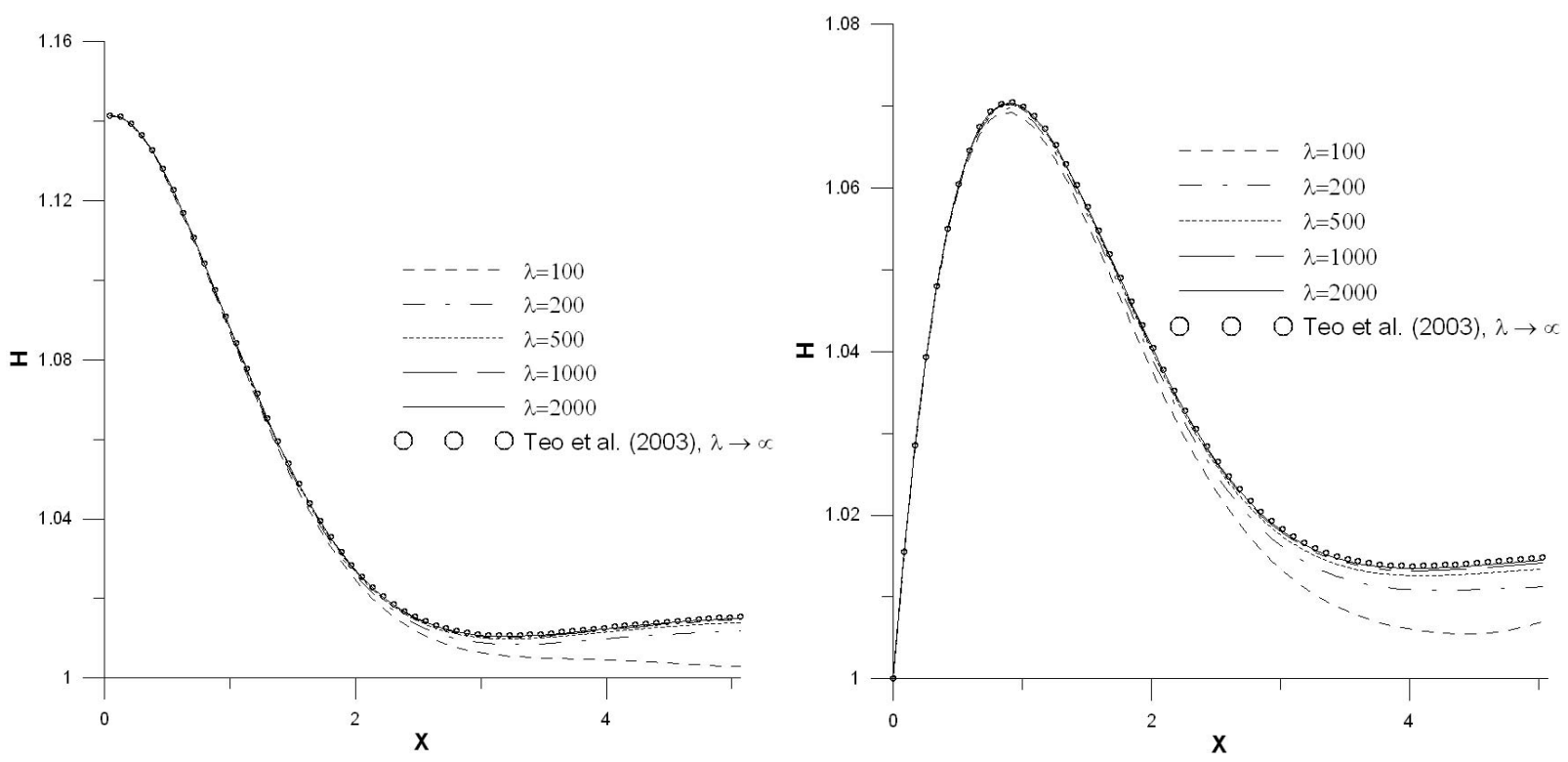

Fig. 3. Comparison between tide-induced water table level $(H)$ in Teo et al. (2003) and the present solution for various length $(\lambda)$ of the oceanic island $\left(\varepsilon=0.3, \alpha=0.2, \beta_{1}=\beta_{2}=45^{\circ}\right)$ (a) $T=\pi / 4$; (b) $T=\pi / 2$.

search, Inc., 2007). Similarly, for the special case of semihalf costal aquifer, Eq. (30) is identical to the solutions in Teo et al. (2003) for first-order approximation in $\varepsilon$ for order $\alpha^{2}$.

\subsubsection{Second-order $\left(O\left(\varepsilon^{2}\right)\right)$ approximation:}

At $O\left(\varepsilon^{2}\right), H_{2}$ is expanded to

$H_{2}=\sum_{n=1}^{\infty} \alpha^{n} H_{2 n}$

Consequently, the equation and boundary conditions in Eq. (21) is further adapted to

$O\left(\varepsilon^{2} \alpha^{1}\right): 2 H_{21 T_{1}}=H_{21 X_{1} X_{1}}+\frac{1}{3} H_{01 X_{1} X_{1} X_{1} X_{1}}$

$H_{21}\left(0, T_{1}\right)=H_{21}\left(X_{R}, T_{1}\right)=0$

$O\left(\varepsilon^{2} \alpha^{2}\right): 2 H_{22 T_{1}}+2 \cot \beta_{1} \sin T_{1} H_{11 X_{1}}=H_{22 X_{1} X_{1}}$

$+\left(H_{01} H_{21}\right)_{X_{1} X_{1}}+\frac{1}{3} H_{02 X_{1} X_{1} X_{1} X_{1}}+\left(H_{01} H_{01 X_{1} X_{1}}\right)_{X_{1} X_{1}}$

$H_{22}\left(0, T_{1}\right)=H_{22}\left(X_{R}, T_{1}\right)=0$

The solutions of the boundary value problems shown above are

$$
\begin{aligned}
& H_{21}=\frac{1}{2}\left[g_{1} e^{X_{1}} \cos \left(T_{1}+X_{1}\right)+g_{2} e^{X_{1}} \sin \left(T_{1}+X_{1}\right)\right. \\
& \left.+g_{3} e^{-X_{1}} \cos \left(T_{1}-X_{1}\right)+g_{4} e^{-X_{1}} \sin \left(T_{1}-X_{1}\right)\right] \\
& +\frac{1}{3}\left\{-X_{1} e^{-X_{1}} \cos \left(T_{1}-X_{1}\right)-X_{1} e^{-X_{1}} \sin \left(T_{1}-X_{1}\right)\right.
\end{aligned}
$$

$$
\begin{aligned}
& +\left(a_{1}-a_{2}\right) X_{1}\left[e^{X_{1}} \cos \left(T_{1}+X_{1}\right)+e^{-X_{1}} \cos \left(T_{1}-X_{1}\right)\right] \\
& \left.+\left(a_{1}+a_{2}\right) X_{1}\left[e^{X_{1}} \sin \left(T_{1}+X_{1}\right)+e^{-X_{1}} \sin \left(T_{1}+X_{1}\right)\right]\right\}
\end{aligned}
$$

$$
\begin{aligned}
& H_{22}=k_{1} e^{\sqrt{2} X_{1}} \cos \left(2 T_{1}+\sqrt{2} X_{1}\right)+k_{2} e^{\sqrt{2} X_{1}} \sin \left(2 T_{1}+\sqrt{2} X_{1}\right) \\
& +k_{3} e^{-\sqrt{2} X_{1}} \cos \left(2 T_{1}-\sqrt{2} X_{1}\right)+g_{4} k^{-\sqrt{2} X_{1}} \sin \left(2 T_{1}-\sqrt{2} X_{1}\right) \\
& +\varphi_{1} X_{1} e^{-2 X_{1}} \cos \left(2 T_{1}-2 X_{1}\right)+\varphi_{2} X_{1} e^{-2 X_{1}} \sin \left(2 T_{1}-2 X_{1}\right) \\
& +\varphi_{3} X_{1} e^{2 X_{1}} \cos \left(2 T_{1}+2 X_{1}\right)+\varphi_{4} X_{1} e^{2 X_{1}} \sin \left(2 T_{1}+2 X_{1}\right)
\end{aligned}
$$

Table 1 also shows the coefficients $g_{1}, g_{2}, g_{3}, g_{4}, k_{1}, k_{2}, k_{3}$, $k_{4}, \varphi_{1}, \varphi_{2}, \varphi_{3}$ and $\varphi_{4}$ in Eqs. (33) and (34). The present solutions second-order approximation in $\varepsilon$ for order $\alpha^{1}$ and $\alpha^{2}$ can be reduced to the solutions in Teo et al. (2003) with the same order for a semi-half costal aquifer.

\section{Results and discussion}

Figure 2 shows the distribution of water table level $(H)$ versus time $(T / 2 \pi)$ for various order solutions at the horizontal distance $X=1$ and $\beta_{1}=\beta_{2}=45^{\circ}$. This figure can be used to investigate the effects of the higher-order components on the water table fluctuations in an oceanic island with sloping beaches. In Fig. $2 \mathrm{a}$, the solutions of water table level for $O\left(\alpha^{2}\right), O\left(\varepsilon \alpha^{2}\right)$ and $O\left(\varepsilon^{2} \alpha^{2}\right)$ are close when $\alpha=0.2$ and $\varepsilon=0.3$. As demonstrated in Fig. $2 \mathrm{~b}$ and c, the difference between zero-order and higher-order solutions increases with $\alpha$ or $\varepsilon$ and significant differences are observed in Fig. $2 \mathrm{~d}$ when $\alpha=0.4$ and $\varepsilon=0.5$. 

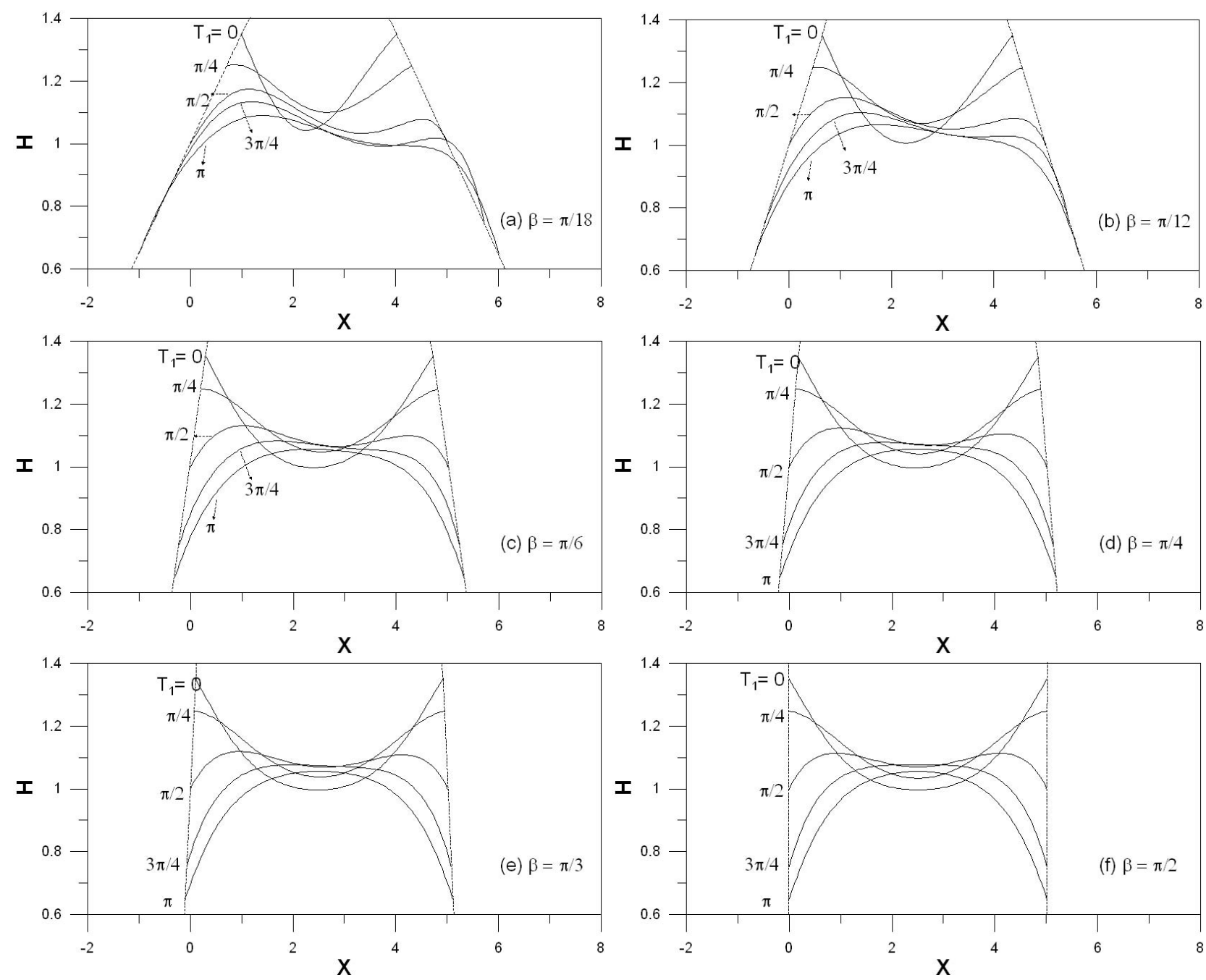

Fig. 4. Distribution of water table level $(H)$ versus horizontal distance $(X)$ for various beach slopes $(\varepsilon=0.5, \alpha=0.35)$.

Comparisons between higher-order solution in Teo et al. (2003) and this study are drawn in Fig. 3a and b, respectively, for $T=\pi / 4$ and $\pi / 2$ with various lengths of the oceanic island. These figures indicate that the present solution is getting close to Teo et al.'s solution as $\lambda$ increases for different times. Therefore, the present solution is applicable for describing the water table level at short horizontal distance in a semi-infinite costal aquifer.

Since the beach slopes may have influence on the water table level, the distributions of water table level versus horizontal distance are illustrated in Fig. 4 for different time with various beach slopes. Graphically, the water table level increases as the beach slope decreases. In other words, the beach slope essentially affects the water table level in oceanic islands. The solution for a semi-half costal aquifer in Teo et al. (2003) is a special case of the present solution when the horizontal distance is not large.
Figure 5a illustrates the differences between second-order $\alpha$ and first-order $\alpha$ approximations for order $\varepsilon^{2}$ when $\alpha=0.2$ and $\varepsilon=0.3$ and $\alpha=0.4$ and $\varepsilon=0.5$. As one can expect, the difference increases with $\alpha$ and $\varepsilon$. Figure $5 \mathrm{~b}$ demonstrates that the differences between second-order $\varepsilon$ and first-order $\varepsilon$ for order $\alpha^{2}$ is smaller than that between and second-order $\varepsilon$ and zero-order $\varepsilon$ for order $\alpha^{2}$ when $T$ is large for both $\alpha=0.2$ and $\varepsilon=0.3$ and $\alpha=0.4$ and $\varepsilon=0.5$.

\section{Concluding remarks}

Using the perturbation technique, an analytical solution is developed for describing the tide-induced groundwater fluctuations in oceanic islands with finite length and different slopes of the beaches. Two perturbation parameters, the shallow water parameter $\varepsilon$ and the amplitude parameter $\alpha$, were used in the present model to derive higher-order solution. 


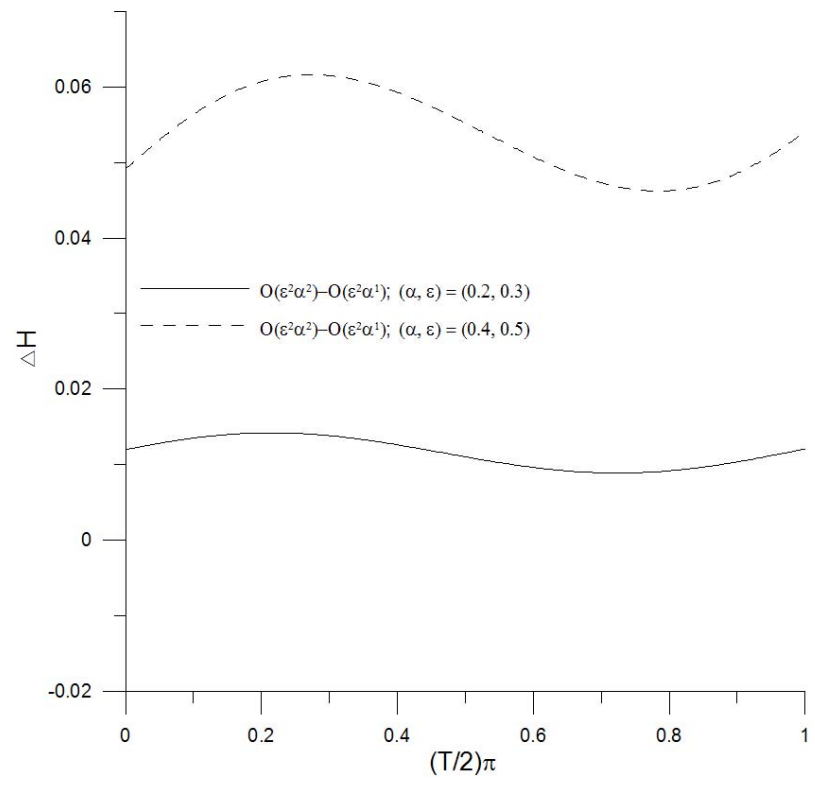

Fig. 5a. Differences between second-order $\alpha$ and first-order $\alpha$ approximations for order $\varepsilon^{2}$ when $(\alpha, \varepsilon)=(0.2,0.3)$ and $(\alpha, \varepsilon)=(0.4$, $0.5)$.

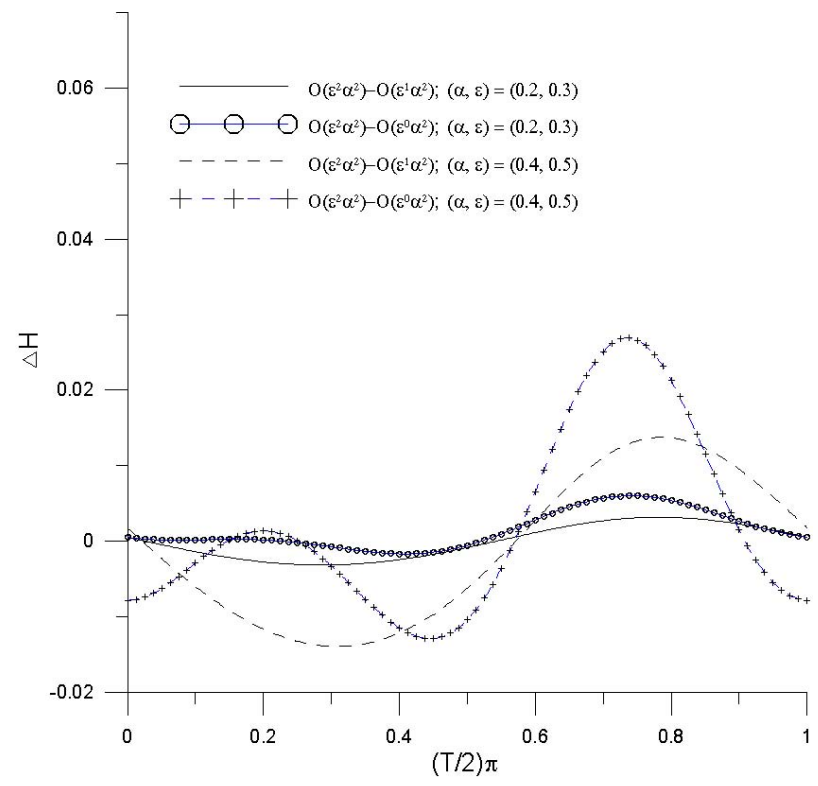

Fig. 5b. Difference between second-order $\varepsilon$, first-order $\varepsilon$ and zeroorder $\varepsilon$ for order $\alpha^{2}$ when $(\alpha, \varepsilon)=(0.2,0.3)$ and $(\alpha, \varepsilon)=(0.4,0.5)$.

The difference between the zero-order and higher-order solution increases with $\varepsilon$ or $\alpha$ and a significant difference is observed when both $\varepsilon$ and $\alpha$ are large. It is found that the beach slopes significantly influence the tide-induced water table and the water table level increases as beach slopes decreases. The present solution is more general than that of Teo et al. (2003) and is capable of describing the case of a infinite coastal aquifer when the horizontal distance is small.

\section{Appendix A}

Substituting Eqs. (18a) and (b) into the governing Eq. (8), boundary conditions in Eqs. (9) and (10) leads to

$$
\begin{aligned}
& \Phi_{0 Z Z}+\varepsilon \Phi_{1 Z Z}+\varepsilon^{2} \Phi_{2 Z Z}+\ldots \\
& =-\varepsilon^{2}\left(\Phi_{0 X_{1} X_{1}}+\varepsilon \Phi_{1 X_{1} X_{1}}+\varepsilon^{2} \Phi_{2 X_{1} X_{1}}+\ldots\right) \\
& \Phi_{0 Z}+\varepsilon \Phi_{1 Z}+\varepsilon^{2} \Phi_{2 Z}+\ldots=0
\end{aligned}
$$

and

$\Phi_{0}+\varepsilon \Phi_{1}+\varepsilon^{2} \Phi_{2}+\ldots=H_{0}+\varepsilon H_{1}+\varepsilon^{2} H_{2}+\ldots$

Equation (A1) can be rearranged in order of $\varepsilon$ as:

$$
\begin{aligned}
& O\left(\varepsilon^{0}\right): \quad \Phi_{0 Z Z}=0 \\
& \Phi_{0 Z}=0 \text { at } Z=0 \\
& \Phi_{0}=H_{0} \text { at } Z=H \\
& O\left(\varepsilon^{1}\right): \quad \Phi_{1 Z Z}=0 \\
& \Phi_{1 Z}=0 \text { at } Z=0 \\
& \Phi_{1}=H_{1} \text { at } Z=H \\
& O\left(\varepsilon^{2}\right): \quad \Phi_{2 Z Z}=-\Phi_{0 X_{1} X_{1}} \\
& \Phi_{2 Z}=0 \text { at } Z=0 \\
& \Phi_{2}=H_{2} \text { at } Z=H
\end{aligned}
$$

Integrating Eq. (A4a) with respect to $Z$ twice obtains

$\Phi_{0}=C_{0}\left(X_{1}, T_{1}\right)+C_{0}^{*} Z$

where $C_{0}$ and $C_{0}^{*}$ are constants of integration. Based on Eqs. (A4b) and (A4c), one has

$\Phi_{0}=H_{0}$

Similarly, we can obtain

$\Phi_{1}=H_{1}$

From Eq. (A6a), one can get

$\Phi_{2}=C_{2}\left(X_{1}, T_{1}\right)+C_{2}^{*} Z-\frac{Z^{2}}{2} \Phi_{0 X_{1} X_{1}}$

Substituting (A6b) into (A10) leads to $C_{2}^{*}=0$ and using (A6c) results in

$H_{2}=C_{2}\left(X_{1}, T_{1}\right)-\frac{H^{2}}{2} \Phi_{0 X_{1} X_{1}}$

and therefore

$\Phi_{2}=H_{2}+\frac{H^{2}}{2} \Phi_{0 X_{1} X_{1}}-\frac{Z^{2}}{2} \Phi_{0 X_{1} X_{1}}$ 
Based on Eq. (15), $\Phi_{T}$ can be expressed as

$\Phi_{T}=\Phi_{T_{1}}+\Phi_{X_{1}} \alpha \varepsilon \cot \beta_{1} \sin T_{1}$

Therefore, Eq. (11) can be expressed as

$2\left(\Phi_{T_{1}}+\Phi_{X_{1}} \alpha \varepsilon \cot \beta_{1} \sin T_{1}\right)=\Phi_{X_{1}}^{2}+\frac{1}{\varepsilon^{2}} \Phi_{Z}^{2}-\frac{1}{\varepsilon^{2}} \Phi_{Z}$

Substituting $\Phi$ in Eqs. (18a), (A8), (A9) and (A12) into (A14) result in the following equation

$$
\begin{aligned}
& 2\left[H_{0 T_{1}}+\varepsilon\left(H_{1 T_{1}}+\alpha \cot \beta_{1} \sin T_{1} H_{0 X_{1}}\right)\right. \\
& \left.+\varepsilon^{2}\left(H_{2 T_{1}}+\alpha \cot \beta_{1} \sin T_{1} H_{1 X_{1}}\right)+\ldots\right] \\
& =\left[H_{0 X_{1}}^{2}+2 \varepsilon H_{0 X_{1}} H_{1 X_{1}}+\varepsilon^{2}\left[H_{1 X_{1}}^{2}+2 H_{0 X_{1}}\left(H_{2 X_{1}}\right.\right.\right. \\
& \left.\left.\left.+H_{0} H_{0 X_{1}} H_{0 X_{1} X_{1}}\right)\right]+\ldots\right]+\frac{1}{\varepsilon^{2}}\left(\varepsilon^{4} H_{0 X_{1} X_{1}}^{2} H_{0}^{2}+\ldots\right) \\
& +\frac{1}{\varepsilon^{2}}\left[\varepsilon^{2} H_{0} H_{0 X_{1} X_{1}}+\varepsilon^{3}\left(H_{1} H_{0 X_{1} X_{1}}+H_{0} H_{1 X_{1} X_{1}}\right)\right. \\
& +\varepsilon^{4}\left(H_{2} H_{0 X_{1} X_{1}}+H_{1} H_{1 X_{1} X_{1}}+H_{0} H_{2 X_{1} X_{1}}\right. \\
& +2 H_{0}^{2} H_{0 X_{1}} H_{0 X_{1} X_{1} X_{1}}+\frac{1}{3} H_{0}^{3} H_{\left.\left.0 X_{1} X_{1} X_{1} X_{1}\right)+\ldots\right]}
\end{aligned}
$$

To obtain Eq. (A15) from Eq. (A14), one also needs to know $\Phi_{3 z}$ and $\Phi_{4 z}$. Then Eq. (A15) can be expressed in terms of different order of $\varepsilon$ as shown in Eqs. (19) to (21).

\section{Appendix B}

The general solution of Eq. (23a) can be expressed as (Bruggeman, 1999)

$$
\begin{aligned}
& H_{01}=\operatorname{Im}\left[\Lambda_{1} \exp \left((1+i) X_{1}\right) \exp \left(i T_{1}\right)\right. \\
& \left.+\Lambda_{1} \exp \left(-(1+i) X_{1}\right) \exp \left(i T_{1}\right)\right]
\end{aligned}
$$

where $\Lambda_{1}$ and $\Lambda_{2}$ are complex numbers. Since the exponential function can be expressed in forms of triangular function, i.e., $\exp (i z)=\cos z+i \sin z$, Eq. (B1) can then be written as

$$
\begin{aligned}
& H_{01}=a_{1} \exp \left(X_{1}\right) \cos \left(T_{1}+X_{1}\right)+a_{2} \exp \left(X_{1}\right) \sin \left(T_{1}+X_{1}\right) \\
& +a_{3} \exp \left(-X_{1}\right) \cos \left(T_{1}-X_{1}\right)+a_{4} \exp \left(-X_{1}\right) \sin \left(T_{1}-X_{1}\right)
\end{aligned}
$$

Substituting the boundary at $X_{1}=0$ in Eqs. (23b) into (B2), we have

$a_{3}=1-a_{1}$

and

$a_{4}=-a_{2}$
Equation (B2) can be therefore expressed as

$$
\begin{aligned}
& H_{01}=e^{-X_{1}} \cos \left(T_{1}-X_{1}\right) \\
& +a_{1}\left[e^{X_{1}} \cos \left(T_{1}+X_{1}\right)-e^{-X_{1}} \cos \left(T_{1}-X_{1}\right)\right] \\
& +a_{2}\left[e^{X_{1}} \sin \left(T_{1}+X_{1}\right)-e^{-X_{1}} \sin \left(T_{1}-X_{1}\right)\right]
\end{aligned}
$$

Expanding the triangular functions in Eq. (B5) and sorting out in terms of sine and cosine functions, the relationships of the coefficients $a_{1}$ and $a_{2}$ can be obtained from the boundary condition of Eq. (23b) at $X_{1}=X_{R}$ as

$2 a_{1} \cos X_{R} \sinh X_{R}+2 a_{2} \sin X_{R} \cosh X_{R}=$

$1-\exp \left(-X_{R}\right) \cos \left(X_{R}\right)$

$-2 a_{1} \sin X_{R} \cosh X_{R}+2 a_{2} \cos X_{R} \sinh X_{R}$

$=-\exp \left(-X_{R}\right) \sin \left(X_{R}\right)$

Furthermore, from Eqs. (B6) and (B7), the coefficients $a_{1}$ and $a_{2}$ can be solved as

$a_{1}=\frac{\cos X_{R}+\exp \left(-X_{R}\right)}{2\left[\cosh X_{R}+\cos X_{R}\right]}$

and

$a_{2}=\frac{\sin X_{R}}{2\left[\cosh X_{R}+\cos X_{R}\right]}$

Based on Eq. (B5), the solution of $H_{01}$ can therefore be expressed in Eq. (25).

Acknowledgements. We are grateful to E. J. M. Veling and another anonymous reviewer for their thoughtful comments and guidance. In addition, research leading to this work has been partially supported by the grants from Taiwan National Science Council under the contract number NSC 96-2221-E-009-087-MY3 and the "Aim for the Top University Plan" of the National Chiao Tung University and Ministry of Education, Taiwan.

Edited by: N. van de Giesen

\section{References}

Batu, V.: Aquifer hydraulics: a comprehensive guide to hydrogeologic data analysis, New York, John Wiley \& Sons Inc., 1998.

Bruggeman, G. A.: Analytical solutions of geohydrological problems, Elsevier Science Ltd, 1999.

Dagan, G.: Second-order theory of shallow free-surface flow in porous media, Quart. J. Mech. Appl. Math., 20, 517-526, 1967.

Freeze, R. A. and Cherry, J. A.: Groundwater, New Jersey, PrenticeHall, Inc., 1979.

Hu, L., Jiao, J. J., and Guo, H.: Analytical studies on transient groundwater flow induced by land reclamation, Water resources research, 44, W11427, doi:10.1029/2008WR006926.

Jiao, J. J., Nandy, S., and Li, H.: Analytical studies on the impact of land reclamation on ground water flow, Ground Water, 39, 912920, 2001. 
Li, L., Barry, D. A., Stagnitti, F., Parlange, J. Y., and Jeng, D. S.: Beach water table fluctuations due to spring-neap tides: moving boundary effects, Adv. Water Resour., 23, 817-824, 2000.

Nielsen, P.: Tidal dynamics of the water table in beaches, Water Resour. Res., 26, 2127-2134, 1990.

Parlange, J. Y., Stagnitti, F., Starr, J. L., and Baddock, R. D.: Freesurface flow in porous media and periodic solution of the shallow flow approximation, J. Hydrol, 70, 251-263, 1984.

Rotzoll, K., El-Kadi, A. I., and Gingerich, S. B.: Analysis of an unconfined aquifer subject to asynchronous dual-tide propagation, Ground Water, 46, 239-250, 2008.
Sun, P., Li, H., Boufadel, M. C., Geng X., and Chen, S.: An analytical solution and case study of groundwater head response to dual tide in an island leaky confined aquifer, Water Resour. Res., 44, W12501, doi:10.1029/2008WR006893.

Teo, H. T., Jeng, D. S., Seymour, B. R., Barry, D. A., and Li, L.: A new analytical solution for water table fluctuations in coastal aquifers with sloping beaches, Adv. Water Resour., 26, 12391247, doi:10.1016/j.advwatres.2003.08.004.

Wolfram Research, Inc., Mathematica, Version 6.0, Champaign, IL, 2007. 